Research Paper

\title{
Variable but consistent pattern of Meningioma 1 gene (MN1) expression in different genetic subsets of acute myelogenous leukaemia and its potential use as a marker for minimal residual disease detection
}

\author{
Sonia Carturan ${ }^{1, *}$, Jessica Petiti, ${ }^{1, *}$ Valentina Rosso ${ }^{1}$, Chiara Calabrese ${ }^{1}$, Elisabetta \\ Signorino ${ }^{1}$, Giada Bot-Sartor ${ }^{1}$, Paolo Nicoli ${ }^{1}$, Daniela Gallo ${ }^{1}$, Enrico Bracco ${ }^{2}$, \\ Alessandro Morotti ${ }^{1}$, Cristina Panuzzo ${ }^{1}$, Enrico Gottardi ${ }^{1}$, Francesco Frassoni ${ }^{3, \#}$, \\ Giuseppe Saglio", ${ }^{1, \#}$, Daniela Cilloni ${ }^{1}$ \\ ${ }^{1}$ Department of Clinical and Biological Sciences, University of Turin, Turin, Italy \\ ${ }^{2}$ Department of Oncology, University of Turin, Turin, Italy \\ ${ }^{3}$ Department of Pediatric Hemato-Oncology and Stem Cell and Cellular Therapy Laboratory, Institute G. Gaslini, Genova, \\ Italy \\ *These author have contributed equally to this work \\ \#These author have contributed equally to this work \\ Correspondence to: Daniela Cilloni, email: daniela.cilloni@unito.it
}

Keywords: minimal residual disease, meningioma 1 gene, acute leukemias, molecular marker

Received: April 21, $2016 \quad$ Accepted: September 14, $2016 \quad$ Published: September 27, 2016

\section{ABSTRACT}

Meningioma 1 (MN1) gene overexpression has been reported in acute myeloid leukaemia (AML) patients and identified as a negative prognostic factor. In order to characterize patients presenting gene overexpression and to verify if $M$ N1 transcript could be a useful marker for minimal residual disease detection, MN1 was quantified in $136 \mathrm{AML}$ patients with different cytogenetic risk and in $\mathbf{5 0}$ normal controls. In 20 patients bearing a fusion gene transcript suitable for minimal residual disease quantitative assessment and in 8 patients with NPM1 mutation, we performed a simultaneous analysis of MN1 and the fusion-gene transcript or NPM1 mutation during follow-up. Sequential MN1 and WT1 analysis was also performed in 13 AML patients lacking other molecular markers. The data obtained show that normal cells consistently express low levels of MN1 transcript. In contrast, high levels of MN1 expression are present in $47 \%$ of patients with normal karyotype and in all cases with inv(16). MN1 levels during follow-up were found to follow the pattern of other molecular markers (fusion gene transcripts, NPM1 and WT1). Increased MN1 expression in the BM during follow up was always found to be predictive of an impending hematological relapse.

\section{INTRODUCTION}

The assessment of minimal residual disease (MRD) has currently become a necessary strategy to better address treatment intensity in acute leukemias [1]. The detection of MRD by RT-PCR is limited to those patients characterized by genetic markers. The latter include fusion genes derived from chromosome translocations, such as $P M L-R A R \alpha A M L 1$ and $C B F-M Y H 11^{2}$ or mutations, for example nucleophosmin (NPM1), [1, 3] which has been validated as a sensitive marker of MRD detection. More recently, studies of next generation sequencing (NGS) allowed to enlarge the spectrum of genetic abnormalities by discovering new mutations and aberrations [4] Basing on these studies, other genetic markers are under investigation, including IDH1 and IDH2 mutations which occur in less than $10 \%$ of the patients[5].

Other genes found overexpressed in AML have been validated for MRD detection in many clinical settings. Among them, one of the most exploited is the Wilms tumor gene ( WT1) [6-8].

Studies of NGS clearly showed that in AML at diagnosis there is a founding clone which prevails and several small subclones, characterized by different mutations, 
which are often undetectable by Sanger sequencing. These subclones can be selected by chemotherapy or by molecular targeted therapies and expand over time thus generating chemoresistance or relapse [4, 9].

Many studies suggest that WT1, although not associated with a specific leukemic clone, is very sensitive in the detection of the persistence or of the reappearance of the disease [8] The fact that its expression is not related to specific genetic alterations allows WT1 to monitor the kinetic of the leukemic cells. Since WT1 is not overexpressed only in AML but in other hematological malignancies including myelodysplastic syndromes [10] and myeloproliferative disorders, [11] it could be considered a "universal marker" of clonal hematopoiesis. Despite the fact that the majority of AML at diagnosis overexpresses $W T 1$, in about $20-30 \%$ of AML the gene is not significantly overexpressed [8]. We therefore explored the possibility of additional molecular markers to monitor the disease.

The meningioma 1 gene (MN1), located on chromosome $22 \mathrm{q} 11$, was cloned from a patient affected by meningioma characterized by the translocation $\mathrm{t}(4 ; 22)$ (p16;q11) [12]. Additional studies identified the fusion between TEL and MN1 genes in AML patients with translocations $\mathrm{t}(12 ; 22)$ (p13;q11) [13]. This genetic alteration, although very rare, represents a relevant prognostic factor with a negative impact on survival. Despite the role of this fusion transcript, it was shown that $M N 1$ overexpression represents a negative prognostic factor in terms of disease free survival [14].

Heuser and colleagues [14] investigated the significance of MN1 expression in a uniformly treated cohort of adult AML patients with normal karyotype. In this study the prognostic relevance of $M N 1$ was compared to other prognostic factors such as FLT3 internal tandem duplication (ITD), MLL and NPM1 mutations. This study suggests that $M N 1$ overexpression is an independent prognostic marker in AML with normal karyotype and it is associated with shorter relapse free survival (RFS) and shorter overall survival (OS) [14].

The two main objects of the present study were the identification and characterization of the subset of patients showing $M N 1$ overexpression and the validation of $M N 1$ as a marker for MRD detection.

\section{RESULTS}

\section{MN1 expression in AML patients at diagnosis}

The expression levels of $M N 1$ transcript in normal controls and in leukemia samples at diagnosis are summarized in Table 1, 2 and Figure 1. The MN1 levels were very low in normal samples: the mean copy number of $M N 1 / 10^{4} A B L$ copies is $130 \pm 94$ (median 136; range 9-300) in peripheral blood (PB) and $285 \pm 117$ in BM (median 254, range 80-500).
Similarly, low levels of expression were detected in normal CD34+ cells obtained from healthy volunteers: mean $M N 1$ copies $/ 10^{4} A B L$ copies $223 \pm 56$ (median 215 copies/10 $A B L$ copies, range 149-300).

Conversely, as shown in Table 1 and 2, 47\% of the samples collected at diagnosis from AML patients characterized by a normal karyotype showed abnormal expression of the MN1 gene. In this subset of patients, the mean value of expression evaluated for 37 out of 79 BM samples showed a transcript amount above the upper limit of normal controls is $9707 \pm 16590$ copies $/ 10^{4} \mathrm{ABL}$ copies (median 5136, range 852-90230). Interestingly, as shown in Figure 1, NK AML and CBF AML seem to segregate in two groups, one with normal values (below $500 \mathrm{MN1}$ copies/104 ABL copies for BM and $300 \mathrm{MN1}$ copies for PB), the second with $M N 1$ values above 1000 copies. This raises the possibility of a "gray zone" between positivity and negativity. At present we cannot establish the prognostic significance of $M N 1$ with values falling in that range.

In accordance, 9 out of 19 PB samples presented abnormal expression with a mean value of $7125 \pm 4663$ (median 6780, range 1367-15900). All samples carrying the fusion transcript $C B \beta-M Y H 11$ expressed a significantly higher amount of $M N 1$ transcript. The mean copy number is $44270 \pm 26285$ (median 46950, range 2149-98000) in $\mathrm{BM}$ and 35200 \pm 21771 (median 34500, range 1400-67999) in PB. These values are significantly higher as compared to controls $(\mathrm{p}<0.0001$ in both $\mathrm{BM}$ and $\mathrm{PB})$. Fifty $\%$ of the samples characterized by the fusion gene RUNX1$A M L 1$ abnormally expressed $M N 1$. The mean value of MN1 copies calculated for BM cells with $M N 1$ expression above the upper limit established by normal samples was $17848 \pm 10925$ (median 16950, range 3500-34000). All four PB samples tested presented high $M N 1$ values with a mean copy number of $16052 \pm 26665$ (median 3475, range 1260-56000). Additionally, four AML patients with sporadic abnormalities such a $\mathrm{t}(9 ; 22)$, trisomy $9,5 \mathrm{q}-$, and complex karyotype were included. All expressed abnormal MN1 transcript values (data shown in Table 1). Finally, the Acute Promyelocytic Leukaemia (APL) samples expressed $M N 1$ values comparable to those of healthy subjects in both BM $(p=0.4)$ and PB $(p=0.08)$.

Interestingly, the paired analysis of $47 \mathrm{~PB}$ and BM samples collected from the same cohort of patients allowed us to establish a remarkable correlation between MN1 expression in PB and BM. Regression analysis provided an $r$ value of 0.91 (Figure 2).

Stratification of patients according to the presence of FLT3 mutation or internal tandem duplication (ITD) demonstrated no significant association between the two abnormalities. MN1 was overexpressed in $35 \%$ of patients with FLT3 ITD, 33\% of patients with the D835 mutation and $50 \%$ of those with wild type FTL3.

Finally, in contrast to previously published data, we were unable to find any significant correlation between 
Table 1: $M N 1$ expression in normal and $A M L$ samples. $B M=$ bone marrow, $P B=$ peripheral $b l o o d ~ N V=$ not valuable, $\mathrm{SD}=$ standard deviation, $\mathrm{CTRL}=$ healthy control

\begin{tabular}{|c|c|c|c|c|c|c|c|}
\hline & \multirow[b]{2}{*}{$\begin{array}{l}\text { Cytogenetic } \\
\text { group }\end{array}$} & \multirow[b]{2}{*}{$\begin{array}{l}\text { Type of } \\
\text { samples }\end{array}$} & \multirow[b]{2}{*}{$\begin{array}{c}\text { No. of samples } \\
\text { tested }\end{array}$} & \multirow{2}{*}{$\begin{array}{c}\text { No. and } \\
\text { percentage } \\
\text { of patients } \\
\text { with MN1 } \\
\text { overexpression }\end{array}$} & \multicolumn{3}{|c|}{ MN1 copies $/ 10^{4}$ ABL copies } \\
\hline & & & & & Mean value \pm SD & Median value & range \\
\hline \multirow[t]{4}{*}{ CTRL } & & $\mathrm{BM}$ & 20 & & $285 \pm 117$ & 254 & $80-500$ \\
\hline & & PB & 30 & & $130 \pm 94$ & 136 & $9-300$ \\
\hline & & CD34+ & 6 & & $223 \pm 56$ & 215 & $149-300$ \\
\hline & TOTAL & & 56 & & & & \\
\hline \multirow[t]{13}{*}{ AML } & $\begin{array}{c}\text { normal } \\
\text { karyotype }\end{array}$ & $\mathrm{BM}$ & 79 & $37(47 \%)$ & $9766 \pm 16590$ & 5136 & $852-90230$ \\
\hline & & PB & 19 & $9(47 \%)$ & $7125 \pm 4663$ & 6780 & $1367-15900$ \\
\hline & $\mathrm{t}(15 ; 17)$ & $\mathrm{BM}$ & 25 & $0(0 \%)$ & $129 \pm 49$ & 130 & $25-219$ \\
\hline & & PB & 7 & $0(0 \%)$ & $99 \pm 75$ & 95 & $20-250$ \\
\hline & $\operatorname{inv}(16)$ & $\mathrm{BM}$ & 16 & $16(100 \%)$ & $44270 \pm 26285$ & 46950 & $2149-98000$ \\
\hline & & $\mathrm{PB}$ & 6 & $6(100 \%)$ & $35200 \pm 21771$ & 34500 & $1400-67999$ \\
\hline & $\mathrm{t}(8 ; 21)$ & $\mathrm{BM}$ & 12 & $6(50 \%)$ & $17848 \pm 10925$ & 16950 & $3500-34000$ \\
\hline & & PB & 4 & $4(100 \%)$ & $16052 \pm 26665$ & 3475 & $1260-56000$ \\
\hline & complex K & $\mathrm{BM}$ & 1 & $1(100 \%)$ & 21080 & NV & NV \\
\hline & $\mathrm{t}(9 ; 22)$ & $\mathrm{BM}$ & 1 & $1(100 \%)$ & 9860 & NV & NV \\
\hline & trysomy 9 & $\mathrm{BM}$ & 1 & $1(100 \%)$ & 8770 & NV & NV \\
\hline & $5 q-$ & $\mathrm{BM}$ & 1 & $1(100 \%)$ & 45935 & NV & NV \\
\hline & TOTAL & & 172 & & & & \\
\hline
\end{tabular}

Table 2: Clinical characteristics of the patients enrolled in the study. Treatment, indicated as A, B, C and D, is described in the "materials and methods" section

\begin{tabular}{|c|c|c|c|c|c|c|c|c|c|}
\hline UPN & age & $\operatorname{sex}$ & cytogenetic & NPM1 & FLT3 & treatment & $\begin{array}{c}M N 1 \\
\text { copies/10000 } \\
\text { ABL copies } \\
\text { in } \mathrm{BM}\end{array}$ & $\begin{array}{c}W T 1 \\
\text { copies/10000 } \\
\text { ABL copies } \\
\text { in } \mathrm{BM}\end{array}$ & $\begin{array}{c}\text { NPM1 } \\
\text { copies } / 10000 \\
\text { ABL copies }\end{array}$ \\
\hline 1 & 22 & $\mathrm{M}$ & NK & $\mathrm{y}$ & $\mathrm{N}$ & A & 199 & 156 & 10230 \\
\hline 2 & 36 & $\mathrm{M}$ & NK & $\mathrm{y}$ & Y & A & 15900 & 3688 & 1860 \\
\hline 3 & 66 & $\mathrm{~F}$ & NK & $\mathrm{y}$ & $\mathrm{Y}$ & B & 199 & 270 & 6780 \\
\hline 4 & 70 & $\mathrm{M}$ & NK & $\mathrm{y}$ & $\mathrm{N}$ & $\mathrm{B}$ & 111 & 189 & 7620 \\
\hline 5 & 38 & F & NK & $\mathrm{y}$ & $\mathrm{N}$ & A & 145 & 340 & NA \\
\hline 6 & 44 & $\mathrm{~F}$ & NK & $\mathrm{y}$ & $\mathrm{N}$ & A & 199 & 1560 & 11831 \\
\hline 7 & 49 & $\mathrm{~F}$ & NK & $\mathrm{y}$ & $\mathrm{N}$ & A & 111 & 223 & 4210 \\
\hline 8 & 57 & $\mathrm{~F}$ & $\mathrm{NK}$ & $\mathrm{y}$ & $\mathrm{N}$ & A & 322 & 1700 & NA \\
\hline 9 & 72 & $\mathrm{M}$ & NK & $\mathrm{y}$ & $\mathrm{N}$ & B & 444 & 568 & NA \\
\hline
\end{tabular}

(Continued) 


\begin{tabular}{|c|c|c|c|c|c|c|c|c|c|}
\hline UPN & age & sex & cytogenetic & NPM1 & FLT3 & treatment & $\begin{array}{c}M N 1 \\
\text { copies } / 10000 \\
\text { ABL copies } \\
\text { in } B M\end{array}$ & $\begin{array}{c}W T 1 \\
\text { copies/10000 } \\
\text { ABL copies } \\
\text { in } B M\end{array}$ & $\begin{array}{c}\text { NPM1 } \\
\text { copies/10000 } \\
\text { ABL copies }\end{array}$ \\
\hline 10 & 61 & $\mathrm{M}$ & NK & $\mathrm{y}$ & $\mathrm{Y}$ & $\mathrm{C}$ & 430 & 13400 & 3280 \\
\hline 11 & 19 & $\mathrm{~F}$ & NK & $\mathrm{y}$ & $\mathrm{N}$ & A & 90230 & 13200 & 8800 \\
\hline 12 & 28 & M & NK & $\mathrm{y}$ & $\mathrm{N}$ & A & 10240 & 3400 & NA \\
\hline 13 & 32 & M & NK & $\mathrm{y}$ & $\mathrm{Y}$ & A & 50 & 450 & 12555 \\
\hline 14 & 45 & M & NK & $\mathrm{y}$ & $\mathrm{N}$ & A & 3570 & 22 & NA \\
\hline 15 & 31 & M & NK & $\mathrm{y}$ & $\mathrm{Y}$ & A & 56000 & 3240 & 18340 \\
\hline 16 & 60 & $\mathrm{~F}$ & NK & $\mathrm{y}$ & $\mathrm{N}$ & A & 11100 & 390 & 2102 \\
\hline 17 & 66 & $\mathrm{M}$ & NK & $\mathrm{y}$ & $\mathrm{Y}$ & B & 14637 & 12 & NA \\
\hline 18 & 73 & $\mathrm{~F}$ & NK & $\mathrm{y}$ & $\mathrm{N}$ & B & 265 & 590 & NA \\
\hline 19 & 74 & $\mathrm{~F}$ & NK & $\mathrm{y}$ & $\mathrm{N}$ & B & 300 & 200 & 9090 \\
\hline 20 & 29 & $\mathrm{~F}$ & NK & $\mathrm{y}$ & $\mathrm{N}$ & A & 1453 & 37 & 15910 \\
\hline 21 & 18 & M & NK & $\mathrm{y}$ & $\mathrm{Y}$ & A & 111 & 11 & NA \\
\hline 22 & 26 & $\mathrm{M}$ & NK & $\mathrm{y}$ & $\mathrm{N}$ & A & 2450 & 2350 & 2230 \\
\hline 23 & 29 & $\mathrm{~F}$ & NK & $\mathrm{y}$ & $\mathrm{N}$ & A & 4500 & 8090 & NA \\
\hline 24 & 38 & $\mathrm{~F}$ & NK & $\mathrm{y}$ & $\mathrm{N}$ & A & 5080 & 9030 & NA \\
\hline 25 & 54 & M & NK & $\mathrm{y}$ & $\mathrm{N}$ & A & 344 & 88 & 13905 \\
\hline 26 & 58 & $\mathrm{M}$ & NK & $\mathrm{y}$ & $\mathrm{N}$ & A & 1460 & 9000 & NA \\
\hline 27 & 73 & $\mathrm{M}$ & NK & $\mathrm{y}$ & $\mathrm{N}$ & B & 2466 & 45 & 17600 \\
\hline 28 & 70 & $\mathrm{~F}$ & NK & $\mathrm{y}$ & $\mathrm{N}$ & B & 8799 & 23 & NA \\
\hline 29 & 48 & $\mathrm{~F}$ & NK & $\mathrm{y}$ & $\mathrm{N}$ & A & 666 & 1900 & NA \\
\hline 30 & 68 & $\mathrm{~F}$ & NK & $\mathrm{y}$ & $\mathrm{Y}$ & B & 156 & 340 & 1190 \\
\hline 31 & 44 & $\mathrm{M}$ & NK & $\mathrm{y}$ & $\mathrm{N}$ & A & 468 & 1200 & NA \\
\hline 32 & 41 & $\mathrm{M}$ & NK & $\mathrm{y}$ & $\mathrm{N}$ & A & 2566 & 18 & 220 \\
\hline 33 & 50 & $\mathrm{~F}$ & NK & $\mathrm{y}$ & $\mathrm{N}$ & A & 243 & 2510 & NA \\
\hline 34 & 74 & $\mathrm{M}$ & NK & $\mathrm{y}$ & $\mathrm{N}$ & B & 222 & 2560 & NA \\
\hline 35 & 73 & $\mathrm{~F}$ & NK & $\mathrm{y}$ & $\mathrm{N}$ & B & 1890 & 40 & 80279 \\
\hline 36 & 30 & $\mathrm{M}$ & NK & $\mathrm{y}$ & $\mathrm{N}$ & A & 1570 & 62 & NA \\
\hline 37 & 49 & $\mathrm{~F}$ & NK & $\mathrm{y}$ & $\mathrm{N}$ & A & 5555 & 5200 & NA \\
\hline 38 & 45 & $\mathrm{M}$ & NK & $\mathrm{y}$ & $\mathrm{N}$ & A & 400 & 250 & 7700 \\
\hline 39 & 27 & $\mathrm{M}$ & NK & $\mathrm{y}$ & $\mathrm{Y}$ & A & 345 & 660 & NA \\
\hline 40 & 69 & $\mathrm{~F}$ & NK & $\mathrm{y}$ & $\mathrm{N}$ & B & 6870 & 66 & NA \\
\hline 41 & 22 & $\mathrm{~F}$ & NK & $\mathrm{y}$ & $\mathrm{N}$ & A & 1790 & 21900 & 2200 \\
\hline 42 & 30 & $\mathrm{M}$ & NK & $\mathrm{y}$ & $\mathrm{N}$ & A & 5666 & 10300 & NA \\
\hline 43 & 68 & $\mathrm{M}$ & NK & $\mathrm{y}$ & $\mathrm{N}$ & B & 3573 & 10 & NA \\
\hline 44 & 65 & $\mathrm{M}$ & NK & $\mathrm{y}$ & $\mathrm{N}$ & B & 1888 & 23200 & 14200 \\
\hline 45 & 61 & $\mathrm{~F}$ & NK & $\mathrm{y}$ & $\mathrm{N}$ & A & 12500 & 8700 & $\begin{array}{c}\text { NA } \\
\text { (Continued) }\end{array}$ \\
\hline
\end{tabular}




\begin{tabular}{|c|c|c|c|c|c|c|c|c|c|}
\hline UPN & age & $\operatorname{sex}$ & cytogenetic & NPM1 & FLT3 & treatment & $\begin{array}{c}M N 1 \\
\text { copies/10000 } \\
\text { ABL copies } \\
\text { in } B M\end{array}$ & $\begin{array}{c}W T 1 \\
\text { copies/10000 } \\
\text { ABL copies } \\
\text { in BM }\end{array}$ & $\begin{array}{c}\text { NPM1 } \\
\text { copies/10000 } \\
\text { ABL copies }\end{array}$ \\
\hline 46 & 45 & $\mathrm{M}$ & NK & $\mathrm{N}$ & $\mathrm{N}$ & A & 120 & 7800 & \\
\hline 47 & 31 & $\mathrm{M}$ & NK & $\mathrm{N}$ & $\mathrm{N}$ & A & 258 & 2140 & \\
\hline 48 & 30 & $\mathrm{~F}$ & NK & $\mathrm{N}$ & $\mathrm{Y}$ & A & 340 & 220 & \\
\hline 49 & 48 & $\mathrm{~F}$ & NK & $\mathrm{N}$ & $\mathrm{N}$ & A & 422 & 900 & \\
\hline 50 & 44 & $\mathrm{~F}$ & NK & $\mathrm{N}$ & $\mathrm{N}$ & A & 254 & 8560 & \\
\hline 51 & 48 & $\mathrm{~F}$ & NK & $\mathrm{N}$ & $\mathrm{N}$ & $\mathrm{C}$ & 18890 & 22 & \\
\hline 52 & 31 & $\mathrm{~F}$ & NK & $\mathrm{N}$ & D835 & A & 299 & 334 & \\
\hline 53 & 64 & $\mathrm{M}$ & NK & $\mathrm{N}$ & $\mathrm{N}$ & A & 154 & 14500 & \\
\hline 54 & 63 & M & NK & $\mathrm{N}$ & $\mathrm{N}$ & $\mathrm{C}$ & 197 & 2230 & \\
\hline 55 & 20 & $\mathrm{~F}$ & NK & $\mathrm{N}$ & $\mathrm{N}$ & A & 15730 & 13200 & \\
\hline 56 & 46 & M & NK & $\mathrm{N}$ & $\mathrm{N}$ & A & 13000 & 78 & \\
\hline 57 & 66 & $\mathrm{~F}$ & NK & $\mathrm{N}$ & $\mathrm{N}$ & B & 312 & 223 & \\
\hline 58 & 33 & M & NK & $\mathrm{N}$ & $\mathrm{Y}$ & A & 311 & 1880 & \\
\hline 59 & 47 & M & NK & $\mathrm{N}$ & $\mathrm{N}$ & A & 4500 & 34 & \\
\hline 60 & 74 & $\mathrm{~F}$ & NK & $\mathrm{N}$ & $\mathrm{N}$ & B & 500 & 890 & \\
\hline 61 & 60 & $\mathrm{~F}$ & NK & $\mathrm{N}$ & $\mathrm{N}$ & $\mathrm{C}$ & 211 & 228 & \\
\hline 62 & 61 & $\mathrm{M}$ & NK & $\mathrm{N}$ & $\mathrm{N}$ & A & 466 & 2460 & \\
\hline 63 & 70 & $\mathrm{M}$ & NK & $\mathrm{N}$ & $\mathrm{N}$ & B & 444 & 2184 & \\
\hline 64 & 22 & $\mathrm{M}$ & NK & $\mathrm{N}$ & $\mathrm{N}$ & A & 499 & 3340 & \\
\hline 65 & 50 & $\mathrm{M}$ & NK & $\mathrm{N}$ & $\mathrm{N}$ & A & 476 & 5510 & \\
\hline 66 & 49 & $\mathrm{~F}$ & NK & $\mathrm{N}$ & $\mathrm{N}$ & $\mathrm{C}$ & 311 & 1990 & \\
\hline 67 & 32 & $\mathrm{~F}$ & NK & $\mathrm{N}$ & $\mathrm{N}$ & A & 6780 & 28 & \\
\hline 68 & 72 & $\mathrm{~F}$ & NK & $\mathrm{N}$ & $\mathrm{N}$ & B & 8900 & 88 & \\
\hline 69 & 44 & $\mathrm{~F}$ & NK & $\mathrm{N}$ & $\mathrm{N}$ & A & 222 & 750 & \\
\hline 70 & 70 & $\mathrm{M}$ & NK & $\mathrm{N}$ & D835 & B & 143 & 145 & \\
\hline 71 & 28 & $\mathrm{M}$ & NK & $\mathrm{N}$ & $\mathrm{N}$ & A & 5136 & 2200 & \\
\hline 72 & 36 & $\mathrm{~F}$ & NK & $\mathrm{N}$ & $\mathrm{N}$ & A & 5305 & 5780 & \\
\hline 73 & 49 & $\mathrm{M}$ & NK & $\mathrm{N}$ & $\mathrm{N}$ & A & 852 & 31 & \\
\hline 74 & 51 & $\mathrm{~F}$ & NK & $\mathrm{N}$ & $\mathrm{N}$ & A & 1674 & 8800 & \\
\hline 75 & 70 & $\mathrm{M}$ & NK & $\mathrm{N}$ & $\mathrm{N}$ & B & 1367 & 3280 & \\
\hline 76 & 66 & $\mathrm{M}$ & NK & $\mathrm{N}$ & D835 & B & 1790 & 1120 & \\
\hline 77 & 42 & $\mathrm{~F}$ & NK & $\mathrm{N}$ & $\mathrm{Y}$ & A & 200 & 676 & \\
\hline 78 & 26 & $\mathrm{~F}$ & NK & $\mathrm{N}$ & $\mathrm{N}$ & A & 210 & 2250 & \\
\hline 79 & 19 & $\mathrm{~F}$ & NK & $\mathrm{N}$ & $\mathrm{N}$ & A & 5680 & 34 & \\
\hline 80 & 56 & $\mathrm{~F}$ & $\mathrm{t}(15 ; 17)$ & $\mathrm{N}$ & $\mathrm{N}$ & $\mathrm{D}$ & 192 & 13450 & \\
\hline
\end{tabular}

(Continued) 


\begin{tabular}{|c|c|c|c|c|c|c|c|c|c|}
\hline UPN & age & sex & cytogenetic & NPM1 & FLT3 & treatment & $\begin{array}{c}M N 1 \\
\text { copies } / 10000 \\
\text { ABL copies } \\
\text { in } B M\end{array}$ & $\begin{array}{c}W T 1 \\
\text { copies/10000 } \\
\text { ABL copies } \\
\text { in } B M\end{array}$ & $\begin{array}{c}\text { NPM1 } \\
\text { copies/10000 } \\
\text { ABL copies }\end{array}$ \\
\hline 81 & 66 & $\mathrm{~F}$ & $\mathrm{t}(15 ; 17)$ & $\mathrm{N}$ & $\mathrm{N}$ & $\mathrm{D}$ & 165 & 23410 & \\
\hline 82 & 34 & $\mathrm{M}$ & $\mathrm{t}(15 ; 17)$ & $\mathrm{N}$ & $\mathrm{N}$ & $\mathrm{D}$ & 177 & 7850 & \\
\hline 83 & 51 & M & $\mathrm{t}(15 ; 17)$ & $\mathrm{N}$ & $\mathrm{N}$ & D & 219 & 8400 & \\
\hline 84 & 58 & M & $\mathrm{t}(15 ; 17)$ & $\mathrm{N}$ & $\mathrm{N}$ & D & 200 & 29800 & \\
\hline 85 & 38 & M & $\mathrm{t}(15 ; 17)$ & $\mathrm{N}$ & $\mathrm{N}$ & $\mathrm{D}$ & 166 & 78400 & \\
\hline 86 & 44 & $\mathrm{~F}$ & $\mathrm{t}(15 ; 17)$ & $\mathrm{N}$ & $\mathrm{Y}$ & D & 130 & 17320 & \\
\hline 87 & 50 & M & $\mathrm{t}(15 ; 17)$ & $\mathrm{N}$ & $\mathrm{N}$ & D & 167 & 54700 & \\
\hline 88 & 39 & $\mathrm{~F}$ & $\mathrm{t}(15 ; 17)$ & $\mathrm{N}$ & $\mathrm{N}$ & $\mathrm{D}$ & 155 & 3300 & \\
\hline 89 & 51 & $\mathrm{~F}$ & $\mathrm{t}(15 ; 17)$ & $\mathrm{N}$ & $\mathrm{N}$ & D & 150 & 7120 & \\
\hline 90 & 49 & M & $\mathrm{t}(15 ; 17)$ & $\mathrm{N}$ & $\mathrm{N}$ & D & 90 & 2650 & \\
\hline 91 & 48 & M & $\mathrm{t}(15 ; 17)$ & $\mathrm{N}$ & $\mathrm{Y}$ & D & 25 & 11070 & \\
\hline 92 & 44 & $\mathrm{~F}$ & $\mathrm{t}(15 ; 17)$ & $\mathrm{N}$ & $\mathrm{N}$ & $\mathrm{D}$ & 122 & 62190 & \\
\hline 93 & 55 & $\mathrm{~F}$ & $\mathrm{t}(15 ; 17)$ & $\mathrm{N}$ & $\mathrm{N}$ & D & 160 & 9240 & \\
\hline 94 & 60 & $\mathrm{~F}$ & $\mathrm{t}(15 ; 17)$ & $\mathrm{N}$ & $\mathrm{N}$ & $\mathrm{D}$ & 150 & 8840 & \\
\hline 95 & 44 & M & $\mathrm{t}(15 ; 17)$ & $\mathrm{N}$ & $\mathrm{N}$ & D & 140 & 3780 & \\
\hline 96 & 49 & $\mathrm{~F}$ & $\mathrm{t}(15 ; 17)$ & $\mathrm{N}$ & $\mathrm{N}$ & $\mathrm{D}$ & 113 & 14200 & \\
\hline 97 & 52 & M & $\mathrm{t}(15 ; 17)$ & $\mathrm{N}$ & $\mathrm{N}$ & D & 80 & 5891 & \\
\hline 98 & 56 & $\mathrm{~F}$ & $\mathrm{t}(15 ; 17)$ & $\mathrm{N}$ & $\mathrm{N}$ & D & 69 & 830 & \\
\hline 99 & 41 & $\mathrm{~F}$ & $\mathrm{t}(15 ; 17)$ & $\mathrm{N}$ & $\mathrm{N}$ & $\mathrm{D}$ & 47 & 41690 & \\
\hline 100 & 65 & $\mathrm{M}$ & $\mathrm{t}(15 ; 17)$ & $\mathrm{N}$ & $\mathrm{N}$ & $\mathrm{D}$ & 76 & 27260 & \\
\hline 101 & 59 & $\mathrm{M}$ & $\mathrm{t}(15 ; 17)$ & $\mathrm{N}$ & $\mathrm{N}$ & D & 98 & 16980 & \\
\hline 102 & 55 & $\mathrm{M}$ & $\mathrm{t}(15 ; 17)$ & $\mathrm{N}$ & $\mathrm{Y}$ & $\mathrm{D}$ & 89 & 27120 & \\
\hline 103 & 45 & $\mathrm{M}$ & $\mathrm{t}(15 ; 17)$ & $\mathrm{N}$ & $\mathrm{N}$ & $\mathrm{D}$ & 100 & 20050 & \\
\hline 104 & 62 & $\mathrm{~F}$ & $\mathrm{t}(15 ; 17)$ & $\mathrm{N}$ & $\mathrm{N}$ & $\mathrm{D}$ & 120 & 15380 & \\
\hline 105 & 49 & $\mathrm{~F}$ & $\mathrm{t}(8 ; 21)$ & $\mathrm{N}$ & $\mathrm{N}$ & A & 34000 & 2180 & 12500 \\
\hline 106 & 21 & $\mathrm{~F}$ & $\mathrm{t}(8 ; 21)$ & $\mathrm{N}$ & $\mathrm{N}$ & A & 3500 & 1650 & 6580 \\
\hline 107 & 68 & $\mathrm{~F}$ & $\mathrm{t}(8 ; 21)$ & $\mathrm{N}$ & $\mathrm{N}$ & B & 9900 & 880 & 2490 \\
\hline 108 & 35 & $\mathrm{M}$ & $\mathrm{t}(8 ; 21)$ & $\mathrm{N}$ & $\mathrm{N}$ & A & 18000 & 1530 & 57810 \\
\hline 109 & 51 & $\mathrm{M}$ & $\mathrm{t}(8 ; 21)$ & $\mathrm{N}$ & $\mathrm{N}$ & A & 25788 & 14300 & 2250 \\
\hline 110 & 66 & $\mathrm{~F}$ & $\mathrm{t}(8 ; 21)$ & $\mathrm{N}$ & $\mathrm{N}$ & B & 15900 & 4300 & 1570 \\
\hline 111 & 32 & $\mathrm{M}$ & $\mathrm{t}(8 ; 21)$ & $\mathrm{N}$ & $\mathrm{N}$ & A & 400 & 910 & 22500 \\
\hline 112 & 56 & $\mathrm{~F}$ & $\mathrm{t}(8 ; 21)$ & $\mathrm{N}$ & $\mathrm{N}$ & A & 340 & 22800 & 4780 \\
\hline 113 & 41 & $\mathrm{M}$ & $\mathrm{t}(8 ; 21)$ & $\mathrm{N}$ & $\mathrm{Y}$ & A & 290 & 18300 & 7630 \\
\hline 114 & 34 & $\mathrm{M}$ & $\mathrm{t}(8 ; 21)$ & $\mathrm{N}$ & $\mathrm{N}$ & A & 350 & 5600 & 10300 \\
\hline 115 & 48 & $\mathrm{M}$ & $\mathrm{t}(8 ; 21)$ & $\mathrm{N}$ & $\mathrm{N}$ & A & 280 & 3490 & 8700 \\
\hline 116 & 68 & $\mathrm{~F}$ & $\mathrm{t}(8 ; 21)$ & $\mathrm{N}$ & $\mathrm{N}$ & B & 380 & 19200 & $\begin{array}{c}22800 \\
\text { (Continued) }\end{array}$ \\
\hline
\end{tabular}




\begin{tabular}{|c|c|c|c|c|c|c|c|c|c|}
\hline UPN & age & $\operatorname{sex}$ & cytogenetic & NPM1 & FLT3 & treatment & $\begin{array}{c}M N 1 \\
\text { copies/10000 } \\
\text { ABL copies } \\
\text { in BM }\end{array}$ & $\begin{array}{c}W T 1 \\
\text { copies/10000 } \\
\text { ABL copies } \\
\text { in BM }\end{array}$ & $\begin{array}{c}N P M 1 \\
\text { copies } / 10000 \\
\text { ABL copies }\end{array}$ \\
\hline 117 & 29 & $\mathrm{~F}$ & $\operatorname{inv}(16)$ & $\mathrm{N}$ & $\mathrm{N}$ & A & 21935 & 750 & 21192 \\
\hline 118 & 59 & $\mathrm{M}$ & $\operatorname{inv}(16)$ & $\mathrm{N}$ & $\mathrm{N}$ & A & 24128 & 3190 & 9027 \\
\hline 119 & 41 & $\mathrm{~F}$ & $\operatorname{inv}(16)$ & $\mathrm{N}$ & $\mathrm{N}$ & A & 72022 & 1840 & 13580 \\
\hline 120 & 42 & $\mathrm{~F}$ & $\operatorname{inv}(16)$ & $\mathrm{N}$ & $\mathrm{N}$ & A & 98000 & 2600 & 8920 \\
\hline 121 & 49 & $\mathrm{~F}$ & $\operatorname{inv}(16)$ & $\mathrm{N}$ & $\mathrm{N}$ & A & 50900 & 2530 & 6510 \\
\hline 122 & 22 & $\mathrm{M}$ & $\operatorname{inv}(16)$ & $\mathrm{N}$ & $\mathrm{N}$ & A & 59000 & 4160 & 62800 \\
\hline 123 & 46 & $\mathrm{~F}$ & $\operatorname{inv}(16)$ & $\mathrm{N}$ & $\mathrm{N}$ & A & 70280 & 3140 & 11519 \\
\hline 124 & 51 & $\mathrm{M}$ & $\operatorname{inv}(16)$ & $\mathrm{N}$ & $\mathrm{N}$ & A & 68900 & 1020 & 37911 \\
\hline 125 & 69 & $\mathrm{M}$ & $\operatorname{inv}(16)$ & $\mathrm{N}$ & $\mathrm{N}$ & B & 28000 & 1010 & 80134 \\
\hline 126 & 39 & $\mathrm{M}$ & $\operatorname{inv}(16)$ & $\mathrm{N}$ & $\mathrm{N}$ & A & 45000 & 47620 & 10100 \\
\hline 127 & 29 & $\mathrm{~F}$ & $\operatorname{inv}(16)$ & $\mathrm{N}$ & $\mathrm{N}$ & A & 27900 & 5610 & 10180 \\
\hline 128 & 60 & $\mathrm{~F}$ & $\operatorname{inv}(16)$ & $\mathrm{N}$ & $\mathrm{N}$ & A & 4700 & 5990 & 12816 \\
\hline 129 & 66 & $\mathrm{~F}$ & $\operatorname{inv}(16)$ & $\mathrm{N}$ & $\mathrm{N}$ & $\mathrm{B}$ & 29804 & 6230 & 11280 \\
\hline 130 & 61 & $\mathrm{M}$ & $\operatorname{inv}(16)$ & $\mathrm{N}$ & $\mathrm{N}$ & A & 56700 & 980 & 29880 \\
\hline 131 & 46 & $\mathrm{~F}$ & $\operatorname{inv}(16)$ & $\mathrm{N}$ & $\mathrm{N}$ & A & 48900 & 4700 & 10065 \\
\hline 132 & 48 & $\mathrm{M}$ & $\operatorname{inv}(16)$ & $\mathrm{N}$ & $\mathrm{N}$ & A & 2149 & 390 & NA \\
\hline 133 & 30 & $\mathrm{~F}$ & complex K & $\mathrm{N}$ & $\mathrm{N}$ & $\mathrm{C}$ & 21080 & 36880 & \\
\hline 134 & 47 & $\mathrm{M}$ & $\mathrm{t}(9 ; 22)$ & $\mathrm{N}$ & $\mathrm{N}$ & A & 9860 & 21370 & \\
\hline 135 & 60 & $\mathrm{M}$ & $46 \mathrm{XY} ;+9$ & $\mathrm{~N}$ & $\mathrm{~N}$ & $\mathrm{C}$ & 8770 & 28600 & \\
\hline 136 & 21 & $\mathrm{~F}$ & $46 X X ;-5 q$ & $\mathrm{~N}$ & $\mathrm{~N}$ & A & 45935 & 990 & \\
\hline
\end{tabular}

EVI-1 and $M N 1$ expression $(\mathrm{r}=0.06)$ or between $M N 1$ and NPM1 mutation $(\mathrm{r}=0.2)$.

\section{MN1 as a target for MRD detection}

To assess the significance of $M N 1$ expression as a marker for MRD detection in AML, the MN1 transcript amount was quantified during follow-up in $20 \mathrm{AML}$ patients characterized by the presence of specific fusiongene transcripts (15 CBF $\beta$-MYH11 and 5 RUNX1-AML1), in 8 patients with NPM1 mutation and in and 13 AML patients (including 3 resistant cases) lacking additional molecular markers but monitored by making use of WT1 quantitative assessment, which we have previously demonstrated to strictly parallel fusion gene transcript behavior [6]. In all cases characterized by the presence of a fusion-gene transcript, the longitudinal pattern of MN1 expression was always found to parallel that of the fusion gene. (Table 3) Three representative AML cases are illustrated in Figure 3. In the inv(16) AML subgroup, the patient who remained in continuous complete remission (CCR) constantly showed $M N 1$ values within the normal range (Table 3), whereas the five patients who ultimately relapsed showed a progressive raising of $M N 1$ levels above the normal range during hematological remission (Table 3 and Figure 3). In the cases illustrated in Figure 3 panel A and C, $M N 1$ values were found above the normal range in concomitance with quite stable values of $C B F \beta$ MYH11 transcript in BM samples taken 3 and 4 months, respectively, before hematological relapse while the patients were still in hematological remission.

Similarly, in the $\mathrm{t}(8 ; 21)$ group, three patients who were in CCR never showed levels of MN1 transcript above the normal range (Table 3), whereas in the two patients who relapsed, increased $M N 1$ levels were detectable 1 and 2 months respectively before the evidence of relapse.

In addition in 8 patients with NPM1 mutation the quantitative analysis of $M N 1$ and NPM1 shows a concordance between the two markers with a progressive increase of both before relapse and normal values of $M N 1$ and negative $N P M 1$ during remission.

Finally, all patients were also monitored using WT1 quantitative assessment. As shown in Figure 3 panel $\mathrm{C}$, and as already demonstrated in our previous 
study and in many studies from the literature, [15] WT1 strictly paralleled the behaviour of the fusion transcripts. Furthermore, we found that MN1 strictly paralleled WT1 in patients without any fusion gene (Figure 4) and in patients with rearrangements in the core binding factor (Figure 3C). Figure 4 shows the two molecular markers used during follow up of a patient who obtained a remission after two courses of chemotherapy and allogeneic bone marrow transplant. In this patient, both markers, WT1 and $M N 1$, returned to normal range and both increased three months before relapse.

Although the presented examples show a good degree of concordance in the curves representing $M N 1$ and fusion genes, they are not completely parallel. In

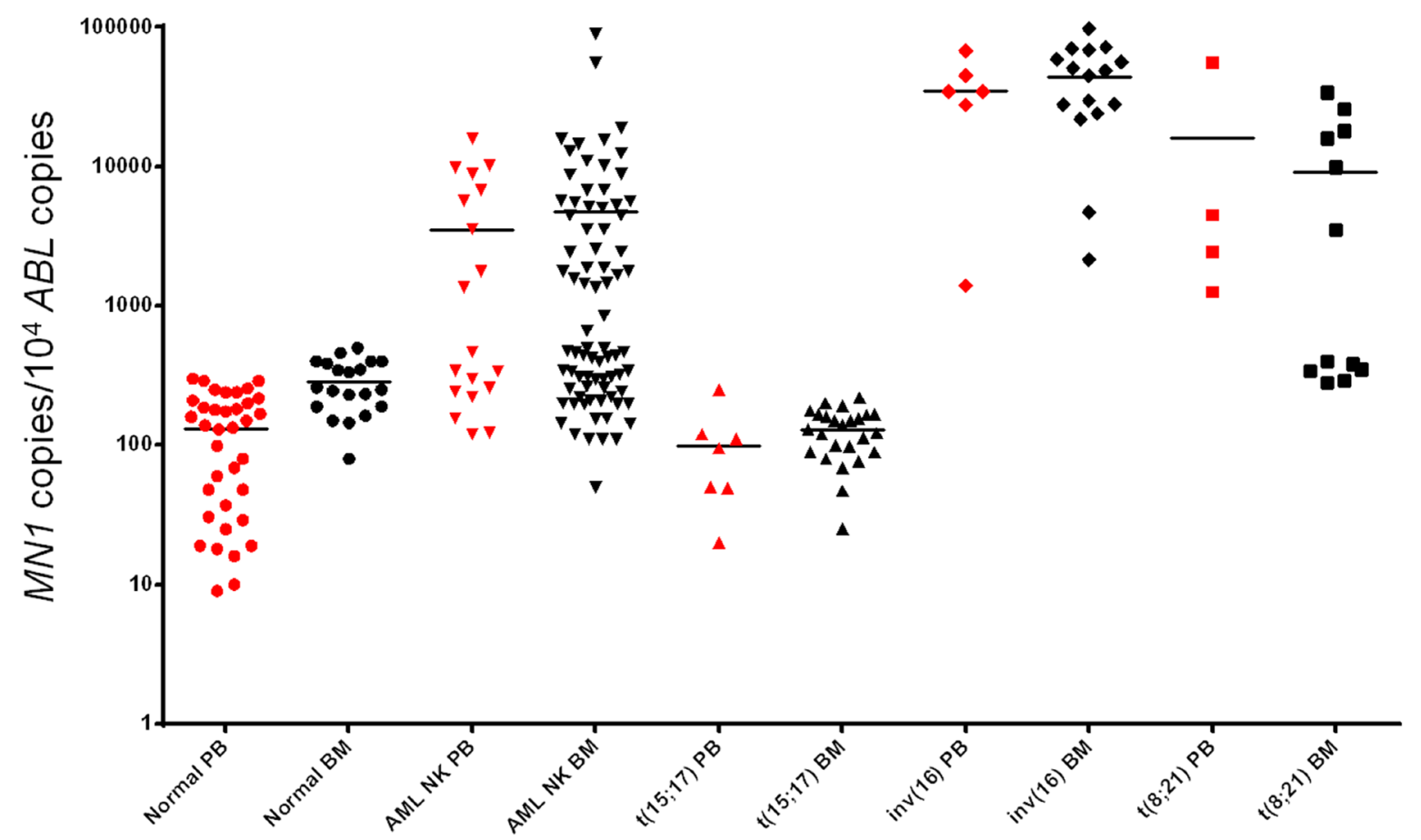

Figure 1: MN1 expression in PB (red dots) and BM (black dots) in samples from healthy volunteers, AML patients with normal karyotype, APL with $\mathrm{t}(15 ; 17)$, AML with inv(16) and AML with $t(8 ; 21)$ chromosomal abnormalities. The transcript amount is expressed as $M N 1$ copies $/ 10^{4} A B L$ copies.

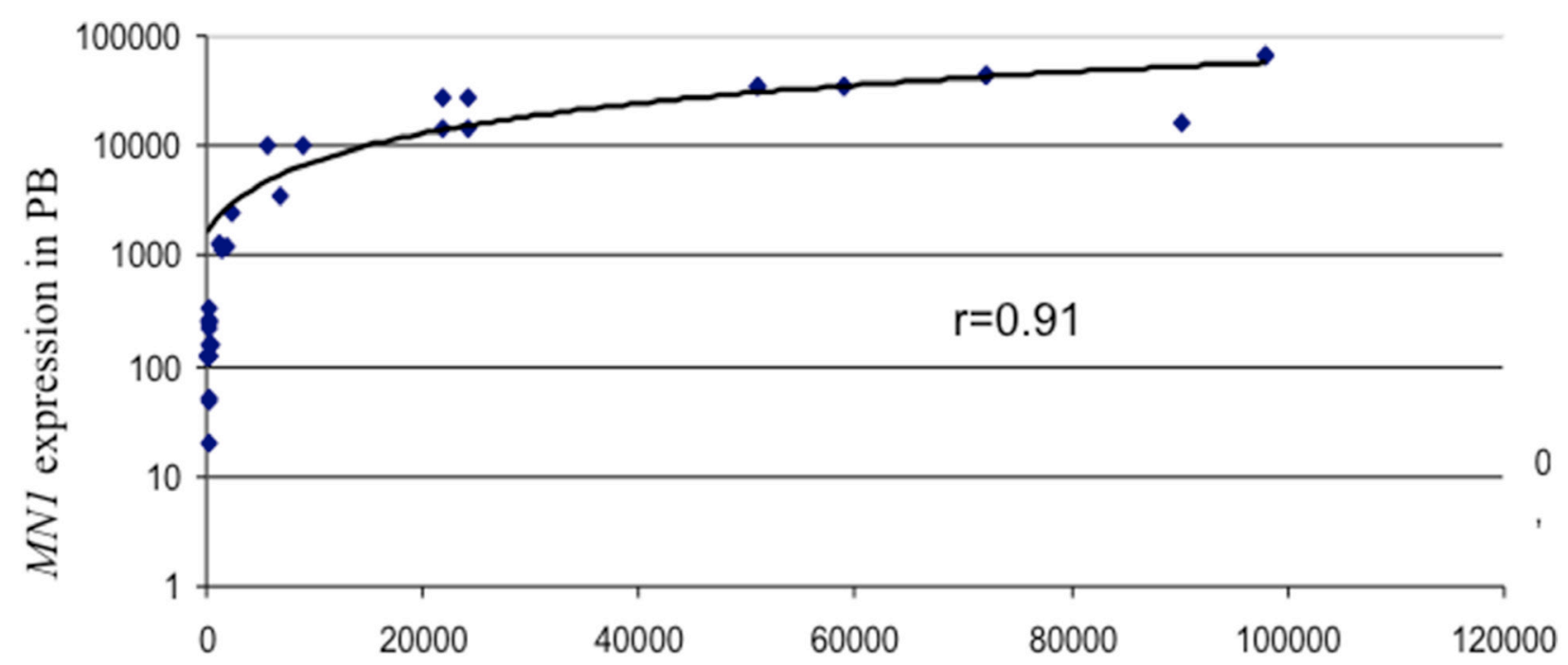

\section{MNI expression in BM}

Figure 2: Correlation between MN1 expression in PB and BM. The transcript amount is expressed as $M N 1$ copies/10 $A B L$ copies. 
Table 3: Simultaneous evaluation of the expression of $M N 1$ and fusion gene transcript (CBF-MYH11 or RUNX1$A M L 1)$ or NMP1 mutation during follow up in patients with AML

\begin{tabular}{|c|c|c|c|c|c|c|c|}
\hline pt & Target gene & Diagnosis & $\begin{array}{c}\text { Post } \\
\text { Induction }\end{array}$ & $\begin{array}{c}\text { CR Post } \\
\text { consolidation I }\end{array}$ & $\begin{array}{c}\text { CR pst Post } \\
\text { consolidation II }\end{array}$ & follow-up & Relapse \\
\hline \multirow[t]{2}{*}{1} & MN1 & 21935 & 210 & 190 & 192 & & \\
\hline & CBF-MYH11 & 21192 & 167 & 12 & 12 & & \\
\hline \multirow[t]{2}{*}{2} & MN1 & 24128 & 87 & 90 & 100 & & \\
\hline & CBF-MYH11 & 9027 & 18 & 9 & 11 & & \\
\hline \multirow[t]{2}{*}{3} & MN1 & 72022 & 312 & 290 & 97 & & \\
\hline & CBF-MYH11 & 13580 & 212 & 21 & 8 & & \\
\hline \multirow[t]{2}{*}{4} & MN1 & 98000 & 95 & 88 & 90 & & \\
\hline & CBF-MYH11 & 8920 & 15 & 21 & 12 & & \\
\hline \multirow[t]{2}{*}{5} & MN1 & 50900 & 320 & 190 & 261 & 190 & \\
\hline & CBF-MYH11 & 6510 & 134 & 67 & 52 & 30 & \\
\hline \multirow[t]{2}{*}{6} & MN1 & 59000 & 110 & 80 & 90 & & \\
\hline & CBF-MYH11 & 62800 & 180 & 200 & 80 & & \\
\hline \multirow[t]{2}{*}{7} & MN1 & 70280 & 88 & 113 & 180 & 883 & 3714 \\
\hline & CBF-MYH11 & 11519 & 340 & 391 & 120 & 312 & 19711 \\
\hline \multirow[t]{2}{*}{8} & MN1 & 68900 & 37 & 41 & 22 & & \\
\hline & CBF-MYH11 & 37911 & 412 & 91 & 11 & & \\
\hline \multirow[t]{2}{*}{9} & MN1 & 28000 & 512 & 193 & 114 & 121 & \\
\hline & CBF-MYH11 & 80134 & 670 & 120 & 69 & 21 & \\
\hline \multirow[t]{2}{*}{10} & MN1 & 45000 & 820 & 632 & 880 & 920 & 53490 \\
\hline & CBF-MYH11 & 10100 & 8 & 4 & 1 & 3 & 9980 \\
\hline \multirow[t]{2}{*}{11} & MN1 & 27900 & 91 & 102 & 100 & 920 & 10142 \\
\hline & CBF-MYH11 & 10180 & 8 & 6 & 1 & 5 & 10090 \\
\hline \multirow[t]{2}{*}{12} & MN1 & 4700 & 66 & 61 & 45 & & \\
\hline & CBF-MYH11 & 12816 & 34 & 12 & 4 & & \\
\hline \multirow[t]{2}{*}{13} & MN1 & 29804 & 97 & 112 & 118 & 121 & 32560 \\
\hline & CBF-MYH11 & 11280 & 8 & 4 & 1 & 4 & 10120 \\
\hline \multirow[t]{2}{*}{14} & MN1 & 56700 & 180 & 134 & 153 & & \\
\hline & CBF-MYH11 & 29880 & 12 & 15 & 12 & & \\
\hline \multirow[t]{2}{*}{15} & MN1 & 48900 & 812 & 410 & 880 & 970 & 10103 \\
\hline & CBF-MYH11 & 10065 & 8 & 6 & 1 & 3 & 10012 \\
\hline pt & Target gene & Diagnosis & $\begin{array}{c}\text { Post } \\
\text { Induction }\end{array}$ & $\begin{array}{c}\text { CR Post } \\
\text { consolidation I }\end{array}$ & $\begin{array}{c}\text { CR pst Post } \\
\text { consolidation II }\end{array}$ & $\begin{array}{l}\text { follow- } \\
\text { up }\end{array}$ & Relapse \\
\hline \multirow[t]{2}{*}{16} & MN1 & 34000 & 211 & 170 & 145 & 161 & \\
\hline & RUNX1-AML1 & 97170 & 4720 & 266 & 27 & 64 & \\
\hline 17 & MN1 & 3500 & 118 & 111 & 131 & 880 & 18670 \\
\hline
\end{tabular}




\begin{tabular}{|c|c|c|c|c|c|c|c|}
\hline pt & Target gene & Diagnosis & $\begin{array}{c}\text { Post } \\
\text { Induction }\end{array}$ & $\begin{array}{c}\text { CR Post } \\
\text { consolidation I }\end{array}$ & $\begin{array}{c}\text { CR pst Post } \\
\text { consolidation II }\end{array}$ & $\begin{array}{l}\text { follow- } \\
\text { up }\end{array}$ & Relapse \\
\hline & RUNX1-AML1 & 9027 & 19 & 7 & 5 & 12 & 8650 \\
\hline \multirow[t]{2}{*}{18} & MN1 & 9900 & 407 & 112 & 109 & & \\
\hline & RUNX1-AML1 & 11830 & 229 & 15 & 2 & & \\
\hline \multirow[t]{2}{*}{19} & MN1 & 18000 & 110 & 141 & 390 & 1020 & 28910 \\
\hline & RUNX1-AML1 & 9902 & 51 & 4 & 3 & 13 & 12500 \\
\hline \multirow[t]{2}{*}{20} & MN1 & 25788 & 99 & 81 & 17 & & \\
\hline & RUNX1-AML1 & 2250 & 22 & 12 & 8 & & \\
\hline pt & Target gene & Diagnosis & $\begin{array}{c}\text { Post } \\
\text { Induction }\end{array}$ & $\begin{array}{c}\text { CR Post } \\
\text { consolidation I }\end{array}$ & $\begin{array}{c}\text { CR pst Post } \\
\text { consolidation II }\end{array}$ & $\begin{array}{c}\text { follow- } \\
\text { up }\end{array}$ & Relapse \\
\hline \multirow[t]{2}{*}{21} & MN1 & 15900 & 113 & 103 & 190 & 1020 & 24500 \\
\hline & NPM1 & 1860 & 21 & 11 & 6 & 54 & 2830 \\
\hline \multirow[t]{2}{*}{22} & MN1 & 90230 & 90 & 81 & 52 & & \\
\hline & NPM1 & 8800 & 21 & 2 & 1 & & \\
\hline \multirow[t]{2}{*}{23} & MN1 & 56000 & 2200 & 860 & 920 & 4290 & 82104 \\
\hline & NPM1 & 18340 & 25 & 28 & 31 & 72 & 11454 \\
\hline \multirow[t]{2}{*}{24} & MN1 & 1453 & 105 & 99 & 92 & & \\
\hline & NPM1 & 15910 & 320 & 76 & 34 & & \\
\hline \multirow[t]{2}{*}{25} & MN1 & 2466 & 990 & 800 & 720 & 1660 & 6792 \\
\hline & NPM1 & 17600 & 120 & 80 & 31 & 60 & 6505 \\
\hline \multirow[t]{2}{*}{26} & MN1 & 2566 & 62 & 71 & 34 & & \\
\hline & NPM1 & 220 & 12 & 2 & 0 & & \\
\hline \multirow[t]{2}{*}{27} & MN1 & 1890 & 63 & 61 & 12 & & \\
\hline & NPM1 & 80279 & 12 & 1 & 1 & & \\
\hline \multirow[t]{2}{*}{28} & MN1 & 1790 & 41 & 44 & 13 & & \\
\hline & NPM1 & 2200 & 5 & 0 & 0 & & \\
\hline \multicolumn{2}{|c|}{ Resistant pt } & rget genes & Diagnosis & Post Induction & & & \\
\hline \multirow[t]{2}{*}{29} & & MN1 & 38910 & 25200 & & & \\
\hline & & WT1 & 8450 & 9240 & & & \\
\hline \multirow[t]{2}{*}{30} & & MN1 & 82100 & 72120 & & & \\
\hline & & WT1 & 7220 & 6123 & & & \\
\hline \multirow[t]{2}{*}{31} & & MN1 & 42193 & 63408 & & & \\
\hline & & WT1 & 9920 & 13450 & & & \\
\hline
\end{tabular}

some cases it seems that $M N 1$ expression, similarly to $W T 1$, is cleared more rapidly than fusion gene transcripts during induction of remission, but it also seems that its elevation is more indicative than the fusion gene transcript in predicting relapse (see Figure 3 panel A and C). At the moment this cannot be easily explained due to our lack of knowledge about the kinetics of $M N 1$ expression in AML.

\section{DISCUSSION}

The negative impact of the persistence of minimal residual disease after chemotherapy or before bone marrow transplantation in acute myeloid leukemia patients is well established [1]. RT-PCR is among the most sensitive methods used for the quantification of the 
A

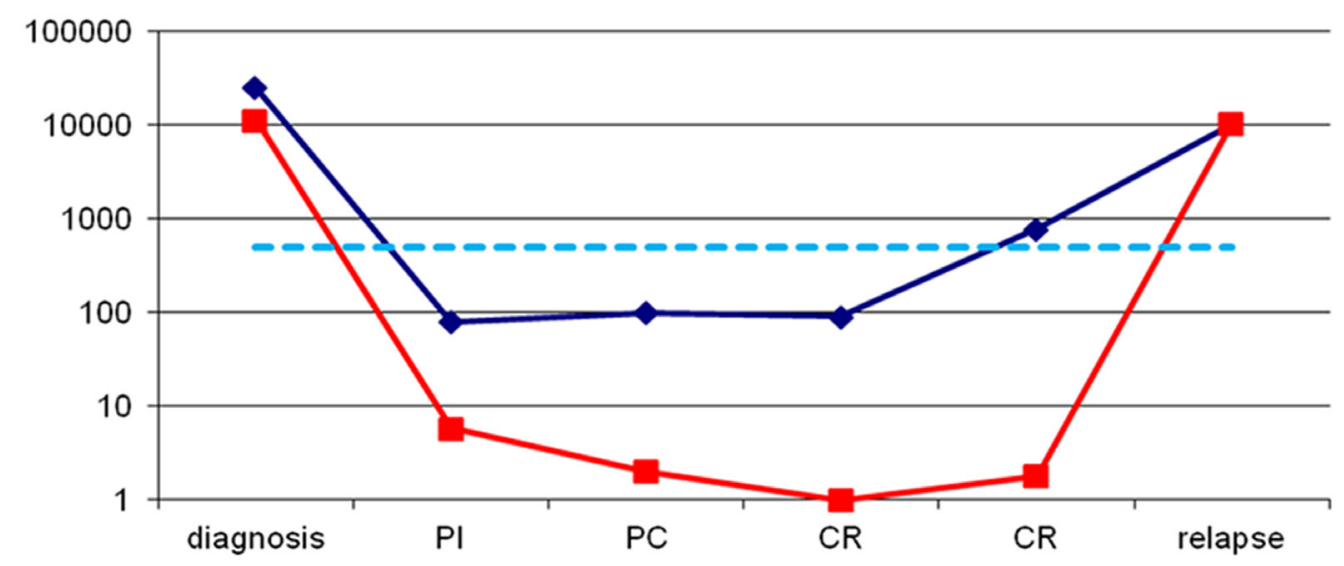

B
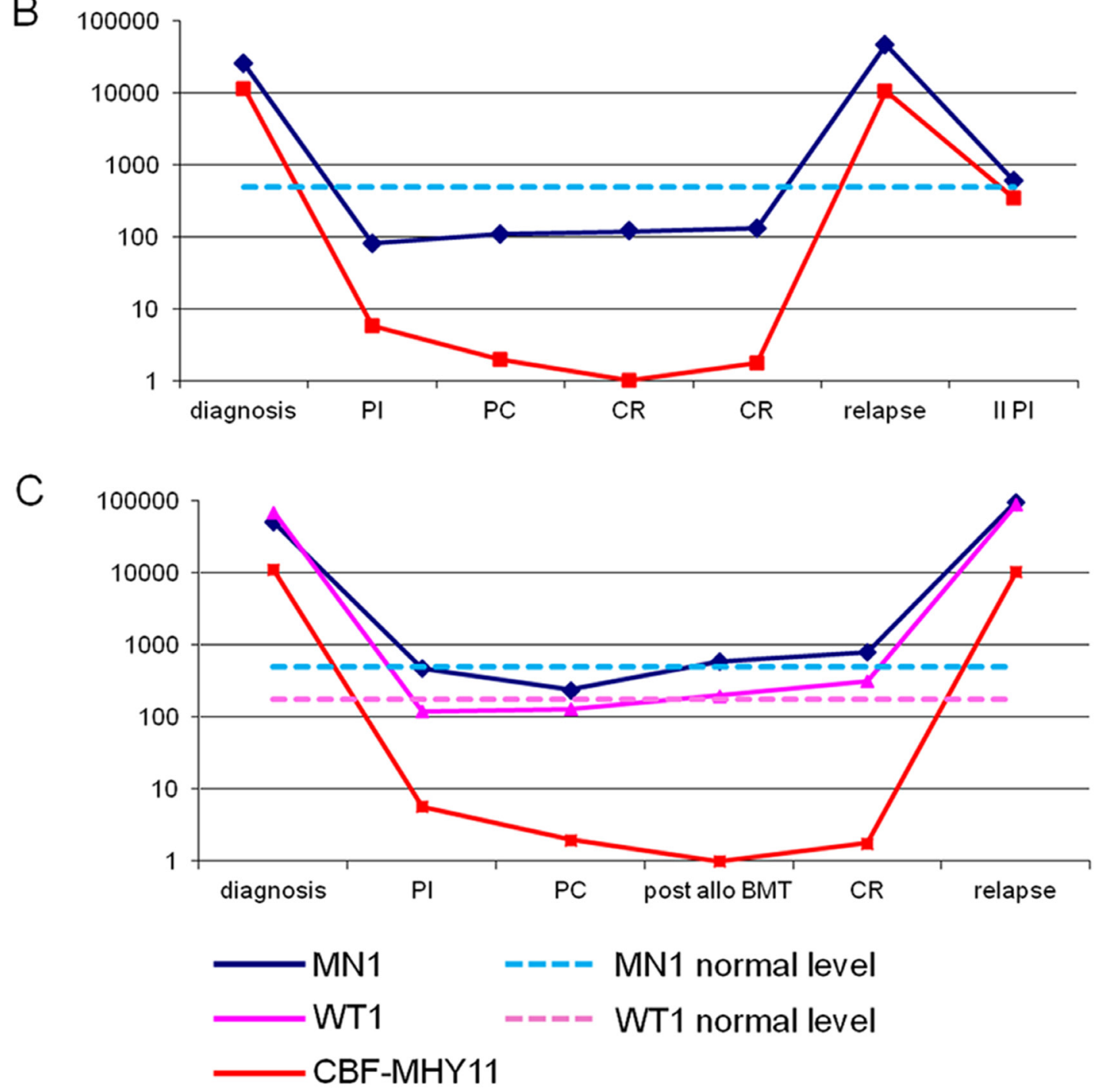

Figure 3: Panel A and B: $M N 1$ transcript expressed as number of copies $/ 10^{4} A B L$ copies (blue line) and $C B F \beta-M Y H 11$ transcript expressed as copy number $/ 10^{4} A B L$ copies (red line) at diagnosis and during follow-up in two patients with inv(16) who relapsed. $M N 1$ increased above the upper normal limit three months before relapse in patient represented in panel A. Panel C: $M N 1$ transcript (blue line), CBFB-MYH1ltranscript (red line) and WT1 transcript (pink line) at diagnosis and during follow-up of a patient with an inv(16) alteration who relapsed after two cycles of chemotherapy and allogeneic bone marrow transplantation (allo BMT). MN1 and WT1 increased above the upper normal limit four months before haematological relapse. $\mathrm{PI}=$ post induction, $\mathrm{PC}=$ post consolidation, $\mathrm{CR}=$ complete remission. 
MRD but it requires the presence of genetic markers in the leukemic clone including fusion transcripts or mutations $[2,3]$.

The disease monitoring became even more cumbersome with the genomic characterization of AML which elegantly demonstrated that, al least at onset, the acute leukemia is mainly constituted by a founding clone with a variable number of mutations and by additional subclones, nearly undetectable, carrying mutations different from the founding clone $[4,9]$.

These subclones might eventually be selected during chemotherapy and expand during the course of the disease $[4,9]$. Considering the dynamic of the leukemic clones it should not be unpopular to suggest the use of a marker not specifically related to a clone but able to identify the presence of leukemic cells independently from their genetic lesions and their phenotype.

In addition, we must consider that in many laboratories NGS technology is not yet available and RQ-PCR targeting all the identified mutations is time consuming and expensive. The advantage of using a single "universal marker" with high sensitivity and specificity allow to better monitor the disease during therapy and in the remission phase.

Interestingly, the association of MN1 with myeloid malignancy goes beyond $M N 1$ involvement in rare translocations such as $\mathrm{t}(12 ; 22)$, as the gene is overexpressed in a significant percentage of AML patients. These has been already demonstrated in literature in some patients characterized by overexpression of the transcription factor ectropic viral integration 1 site (EVII) [16] and in some adult AML patients without karyotypic abnormalities. In the latter case, overexpression of MN1 was associated with a worse prognosis and shorter survival rate [14]. Despite the possibility that $M N 1$ could represent an independent prognostic factor for AML patients, particularly for those with a normal karyotype, there are few data regarding the expression of MN1 in normal hematopoietic cells and in different subtypes of AML. Furthermore, there is currently no evidence that $M N 1$ could represent a suitable marker for minimal residual disease detection. Using a real time quantitative PCR approach, we show that $M N 1$ expression is detectable in all normal bone marrow and peripheral blood samples and CD34 positive cells collected from healthy subjects, although we were able to estimate that normal subjects expressed very low MN1 levels. In contrast, a significant number of patients are characterized by high MN1 transcript amount and, in several cases, the expression is at least 2 or $3 \operatorname{logs}$ higher than controls. It appears that overexpression of MN1 is mainly associated with the inv16 chromosomal abnormality and with a normal karyotype, whereas in $\mathrm{t}(15 ; 17)$ APL the values are consistently comparable to controls. In contrast, we were
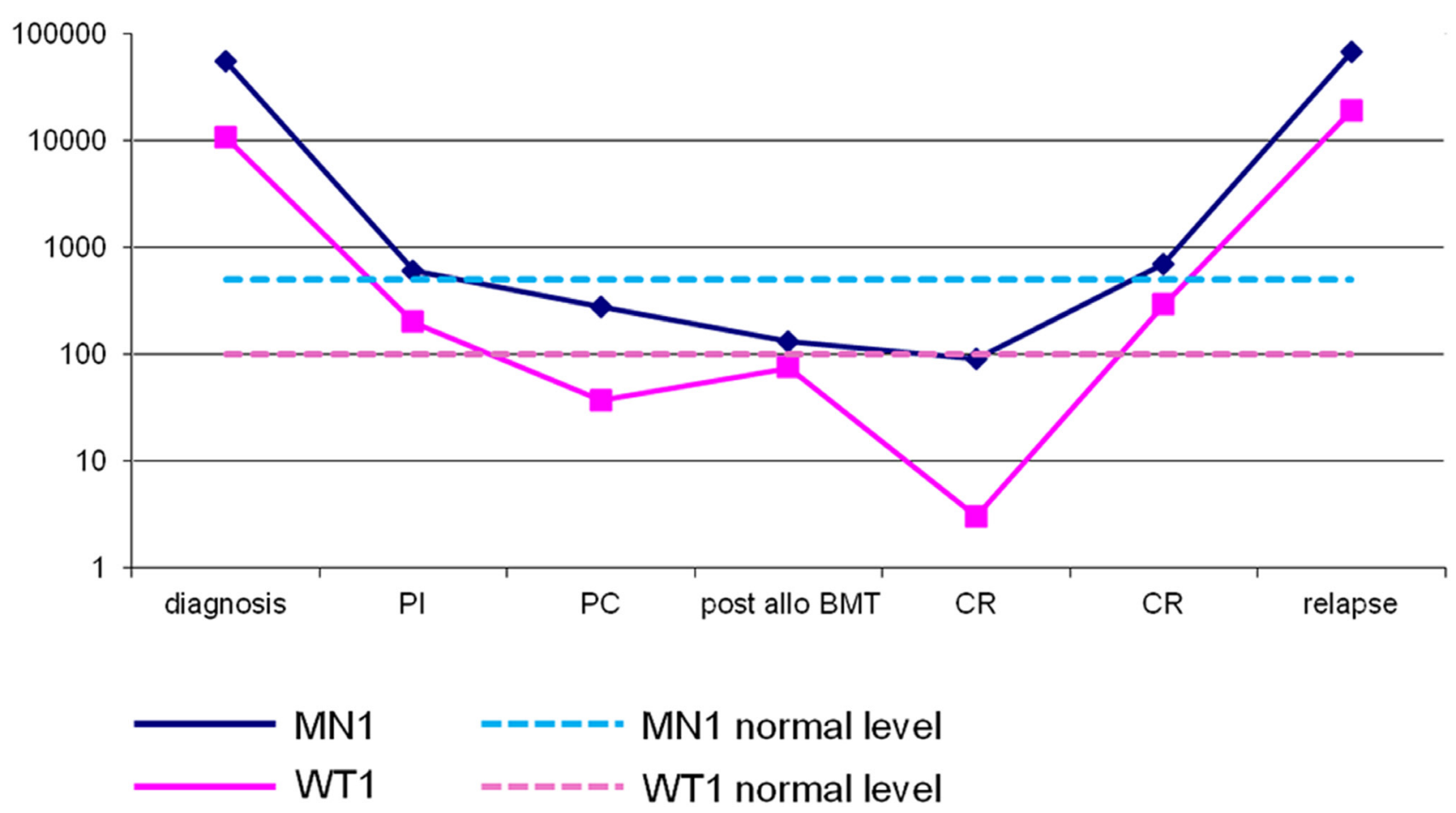

Figure 4: MN1 transcript (blue line) and WT1 transcript (pink line) expressed as number of copies/10 4 ABL copies at diagnosis and during follow-up of a patient with normal karyotype who obtained remission after chemotherapy and allogeneic bone marrow transplant and relapsed six months after transplant. In this patient, both markers, WT1 and $M N 1$, returned to normal range and increased three months before relapse. 
unable to find any significant correlation between FLT3 ITD or mutation or EVI-1 overexpression. Moreover, the finding that CD34-positive cells express low levels of MN1 transcript supports the notion that increased levels of $M N 1$ expression are indeed specific of leukemic blasts and not a simple consequence of the degree of differentiation. Since a significant percentage of AML shows consistently increased MN1 expression levels, this could represent a candidate marker suitable to discriminate between normal and leukemic hematopoiesis and useful to establish the presence, persistence or reappearance of leukemic clone. In particular, our data show that $45 \%$ of AML cases lacking other molecular markers suitable for MRD monitoring express at diagnosis MN1 transcript values above the normal range established by healthy subjects. In this subset of patients, MN1 may represent a reliable marker for MRD detection. So far no data are available concerning the clinical significance of detection of MN1 expression by RT-PCR for monitoring patients with acute leukemia during follow-up. The data presented in this paper show that an accurate quantitative assessment of MN1 transcript amount allows to clearly distinguish between normal and abnormal expression levels of MN1 and, as for $W T 1$, can overcome the problem represented by the minimal amount of gene expression found in normal hematopoietic progenitors. The simultaneous quantitative assessment of the $M N 1$ transcript and of the specific fusion gene or NPM1 mutation showed a good parallelism between the behaviour of the two markers. Indeed, minor discrepancies at low levels of expression of the two markers were observed. In particular, the decrease in MN1 expression seems to be particularly rapid compared to the fusion gene transcript during the induction of remission and its elevation before relapse is more rapid and therefore it is probably more sensitive in predicting relapse. Therefore, even though the degree of sensitivity for MRD detection by analysis of MN1 expression remains to be established, the results obtained show that an increase in $M N 1$ expression above normal levels can be of prognostic significance in predicting relapse during follow-up of AML patients. Although the MN1 gene requires validation as a marker for minimal residual disease in future prospective studies, it seems to be a promising marker for this purpose and further studies should be encouraged.

\section{MATERIALS AND METHODS}

After informed consent, 136 acute myeloid leukaemia patients and 50 healthy volunteers were included in the study. 136 bone marrow samples (BM) and 36 paired peripheral blood samples (PB) were collected from 136 AML patients at diagnosis. In addition, 41 patients were studied during follow-up. The median age was 48 years (range 18-74). All cases were classified according to FAB criteria, characterized at the cytogenetic level by conventional karyotyping and screened by RT-PCR for the presence of the most frequent fusion transcripts, as previously described. ${ }^{2}$ NPM1 mutations ${ }^{3}$ and FLT3 ITD or D835 mutations were screened. WT1 quantitative assessment is available for all samples included in the study and, furthermore, in 40 out of 136 BM samples EVI-1 quantitative assessment was also performed. The FAB distribution was as follows: FAB $\mathrm{M} 0=21, \mathrm{FAB} M 1=22, \mathrm{FAB} M 2=26, \mathrm{FAB} \mathrm{M} 3=25, \mathrm{FAB}$ $\mathrm{M} 4=24, \mathrm{FAB} \mathrm{M} 5=16, \mathrm{FAB} \mathrm{M} 6=2$. Patients younger than 60 years were treated following standard protocols established by the GIMEMA Cooperative Group for the treatment of adult patients with acute myeloid leukemia which included: Induction treatment with a 3-drug regimen: Daunorubicine (DNR) $50 \mathrm{mg} / \mathrm{sqm} /$ day on days 1, 3 and 5; Cytosine-Arabinoside (ARA-C) $100 \mathrm{mg} / \mathrm{sqm} /$ day on days 1 to 10 ; Etoposide $100 \mathrm{mg} / \mathrm{sqm} /$ day on days 1 to 5; to be repeated in case of partial remission (PR). Consolidation therapy with DNR $50 \mathrm{mg} / \mathrm{sqm} /$ day on days 4 to 6 and intermediate-doses ARA-C $(500 \mathrm{mg} / \mathrm{sqm} / 12 \mathrm{~h}$ on days 1 to 6 ) for patients achieving complete remission (CR) after either the first or the second induction cycle.

Additional consolidation treatments with high dose ARA-C were used followed, in high risk patients by allogeneic stem cell transplantation. (This regimen is indicated as treatment $\mathrm{A}$ in Table 2) Elderly or unfit patients were treated with two cycles of daunorubicin 45 $\mathrm{mg} / \mathrm{sqm} /$ day on days 1,3 plus ARA-C $100 \mathrm{mg} / \mathrm{sqm} /$ day on days 1 to 7 followed, in same cases, by autologous stem cell transplantation. (This regimen is indicated as treatment B in Table 2)

Refractory or secondary AML were treated following the Mito-FLAG scheme (Fludarabine $30 \mathrm{mg}$ / sqm day 1-5, ARA-C $2000 \mathrm{mg} / \mathrm{sqm}$ day 1-5, mitoxantrone $7 \mathrm{mg} / \mathrm{sqm}$ day $1,3,5$ and G-CSF $5 \mu \mathrm{g} / \mathrm{kg}$ from day -1) and consolidated as described above. (This regimen is indicated as treatment $\mathrm{C}$ in Table 2). Finally Acute promyelocytic leukemia were treated with anthracyclinebased risk-adapted chemotherapy plus all-trans retinoic acid (ATRA) [17]. (This regimen is indicated as treatment $\mathrm{D}$ in Table 2)

Complete remission was defined according to standard criteria. Finally 30 PB and 20 BM and 6 CD34+ enriched peripheral blood stem cell samples collected from healthy volunteers were included as normal control.

\section{Real time quantitative RT-PCR (RQ-PCR) analysis of MN1 and WT1}

Total RNA was extracted using TRI Reagent solution (Ambion, Waltham, MA USA). Mononuclear cells were separated on a Ficoll-Hypaque density gradient. Total RNA was extracted by standard procedure. The RT (reverse transcription) step was performed as previously described [2, 8]. RQ-PCR reactions and fluorescence measurements were made on the ABI PRISM 7700 
Sequence Detection System (PE Applied Byosystems, Foster City, USA).

Briefly, the RQ-PCR primers and probe for MNI detection were provided by ELItech, Turin, Italy

Primers and probe for $M N 1$ detection are:

5' AGAAGGCCAAACCCCAGAACC-3'

5' GATGGTGAGGCCTTGTTTGCA-3'

5' Fam-ACAGCAAAGAAGCCCAC-MGBNFQ 3'

For $W T 1$ we followed the ELN standardized method reported [8].

The analysis was performed in triplicate and results showing a discrepancy $>1 \mathrm{Ct}$ in one of the wells were excluded and repeated. For quantitative assessment of MN1 a calibration curve with a plasmid containing MN1 target sequences was used (ELItech, Turin, Italy). The MN1 values obtained by RQ-PCR were normalized with respect to the number of $A B L$ transcripts and expressed as $M N 1$ copy number every $10^{4}$ copies of $A B L$. Quantitative assessment of $C B F-M Y H 11$ and $R U N X 1-A M L 1$ transcripts was determined using primers and probes according to standardized procedures. ${ }^{2}$

\section{Real time quantitative RT-PCR (RQ-PCR) analysis of EVI-1}

For $E V I-1$ and $A B L$ quantification, specific assays on demand kits of primers and probe (assay ID for EVI-1 HS01118675 m1 and for ABL Hs00245445 m1 (Applied Byosystems, Foster City, USA) were used following the manufacturer's instructions.

All sample analysis was performed in triplicate and results showing a discrepancy $>1 \mathrm{Ct}$ in one of the wells were excluded and repeated.

EVI-1 Ct obtained by RQ-PCR was normalized with respect to the $\mathrm{Ct}$ of $A B L$ and calibrated with universal RNA (Stratagene, Santa Clara, California, USA) and finally expressed as $2^{-\Delta \Delta \mathrm{Ct}}$.

\section{CD34-positive cells enrichment}

CD34+ cells were enriched according to a magnetic cell sorting methodology (MACS; Miltenyi Biotec, Bergisch Gladbach, Germany).Briefly, mononuclear cells were labeled with a haptenized CD34 antibody (QBEND $\backslash 10)$ that was magnetically labelled in a second step reaction with an anti-hapten antibody coupled to super paramagnetic microbeads. Labelled cells were then separated using a high gradient magnetic separator column placed in a strong magnetic field. The magnetically stained cells were retained in the column, and when the latter was removed from the magnetic field, CD34-positive cells were eluted. At the end of the procedure, CD34 positive cells represented more that $90 \%$ of the total as determined by flow cytometric analysis.

\section{Statistical analysis}

$M N 1$ values obtained for different types of leukemia were compared using the Student's $t$-test.

\section{CONFLICTS OF INTEREST}

The authors declare no conflicts of interest.

\section{GRANT SUPPORT}

DC was funded by grants from: AIRC, MURSTCOFIN,. The study was partially funded by a special grant from "AIRC 5 per mille" to the AGIMM group (AIRC-Gruppo Italiano Malattie Mileoproliferative); for a complete list of AGIMM investigators see at http://www. progettoagimm.it.

FF was supported by Compagnia di San Paolo, Torino and Cinque per mille Istituto Galsini, Ricerca Corrente, Ministero della Salute.

VR is a fellow of Compagnia di San Paolo.

JP is a fellow of Gigi Ghirotti Foundation.

\section{REFERENCES}

1. Ivey A, Hills RK, Simpson MA, Jovanovic JV, Gilkes A, Grech A, Patel Y, BhudiaN, Farah H, Mason J, Wall K, Akiki S, Griffiths M, et al. Assessment of Minimal Residual Disease in Standard-Risk AML. N Engl J Med. 2016; 374:422-433.

2. Gabert J, Beillard E, van der Velden VH, Bi W, Grimwade D, Pallisgaard N, Barbany G, Cazzaniga G, Cayuela JM, Cavé H, Pane F, Aerts JL, De Micheli D, et al. Standardization and quality control studies of 'real-time' quantitative reverse transcriptase polymerase chain reaction of fusion gene transcripts for residual disease detection in leukemia - a Europe Against Cancer program. Leukemia. $2003 ; 17: 2318-2357$.

3. Gorello P, Cazzaniga G, Alberti F, Dell'Oro MG, Gottardi E, Specchia G, Roti G, Rosati R, Martelli MF, Diverio D, Lo Coco F, Biondi A, Saglio G, et al. Quantitative assessment of minimal residual disease in acute myeloidleukemia carrying nucleophosmin (NPM1) gene mutations. Leukemia. 2006; 20:1103-1108.

4. Cancer Genome Atlas Research Network. Genomic and epigenomic landscapes of adult de novo acute myeloid leukemia. N Engl J Med. 2013; 368:2059-2074.

5. Debarri H, Lebon D, Roumier C, Cheok M, MarceauRenaut A, Nibourel O, Geffroy S, Helevaut N, Rousselot P, Gruson B, Gardin C, Chretien ML, Sebda S, et al. IDH1/2 but not DNMT3A mutations are suitable targets for minimal residualdisease monitoring in acute myeloid leukemia patients: a study by the AcuteLeukemia French Association. Oncotarget. 2015; 6:42345-42353. doi: 10.18632/oncotarget.5645. 
6. Cilloni D, Gottardi E, De Micheli D, Serra A, Volpe G, Messa F, Rege-Cambrin G, Guerrasio A, Divona M, Lo Coco F, Saglio G. Quantitative assessment of WT1 expression by real time quantitative PCR may be a useful tool for monitoring minimal residual disease in acute leukemia patients. Leukemia. 2002; 16:2115-2121.

7. Inoue K, Ogawa H, Yamagami T, Soma T, Tani Y, Tatekawa T, Oji Y, Tamaki H, Kyo T, Dohy H, Hiraoka A, Masaoka T, Kishimoto T. Long-term follow-up of minimal residual disease in leukemia patients by monitoring WT1 (Wilms tumor gene) expression levels. Blood. 1996; 88:2267-2278.

8. Cilloni D, Renneville A, Hermitte F, Hills RK, Daly S, Jovanovic JV, Gottardi E, Fava M, Schnittger S, Weiss T, Izzo B, Nomdedeu J, van der Heijden A, et al. Real-time quantitative polymerase chain reaction detection of minimal residual disease by standardized WT1 assay to enhance risk stratification in acute myeloid leukemia: a European Leukemia Net study. J Clin Oncol. 2009; 275:195-201.

9. Grimwade D, Ivey A, Huntly BJ. Molecular landscape of acute myeloid leukemia in younger adults and its clinical relevance. Blood. 2016; 127:29-41.

10. Cilloni D, Gottardi E, Messa F, Fava M, Scaravaglio P, Bertini M, Girotto M, Marinone C, Ferrero D, Gallamini A, Levis A, Saglio G; Piedmont Study Group on Myleodysplastic Syndromes. Significant correlation between the degree of WT1 expression and the International Prognostic Scoring System Score in patients with myelodysplastic syndromes. J Clin Oncol. 2003; 21: 1988-1995.

11. Guglielmelli P, Zini R, Bogani C, Salati S, Pancrazzi A, Bianchi E, Mannelli F, Ferrari S, Le Bousse-Kerdilès MC, Bosi A, Barosi G, Migliaccio AR, Manfredini R, et al. Molecular profiling of $\mathrm{CD} 34+$ cells in idiopathic myelofibrosis identifies a set of disease-associated genes and reveals the clinical significance of Wilms' tumor gene 1 (WT1). Stem Cells. 2007; 25:165-173.
12. Lekanne Deprez RH, Riegman PH, Groen NA, Warringa UL, van Biezen NA, Molijn AC, Bootsma D, de Jong PJ, Menon AG, Kley NA, Seizinger BR, Zwarthoff EC. Cloning and characterization of MN1, a gene from chromosome $22 \mathrm{q} 11$, which is disrupted by a balanced translocation in a meningioma. Oncogene. 1995; 10:1521-1528.

13. Buijs A, Sherr S, van Baal S, van Bezouw S, van der Plas D, Geurts van Kessel A, Riegman P, Lekanne Deprez R, Zwarthoff E, Hagemeijer A, Grosveld G. Translocation $(12 ; 22)(\mathrm{p} 13 ; \mathrm{q} 11)$ in myeloproliferative disorders results in fusion of the ETS-like TEL gene on 12p13 to the MN1 gene on 22q11.Oncogene. 1995; 10:1511-1519. Erratum in: Oncogene. 1995; 11:809.

14. Heuser M, Beutel G, Krauter J, Döhner K, von Neuhoff N, Schlegelberger B, Ganser A. High meningioma 1 (MN1) expression as a predictor for poor outcome in acute myeloid leukemia with normal cytogenetics. Blood. 2006; 108: 3898-3905.

15. Lasa A, Carricondo M, Estivill C, Bussaglia E, Gich I, Brunet S, Aventin A, Sierra J, Nomdedéu JF. WT1 monitoring in core binding factor AML: comparison with specific chimeric products. Leuk Res. 2009; 33:1643-1649.

16. Carella C, Bonten J, Sirma S, Kranenburg TA, Terranova S, Klein-Geltink R, Shurtleff S, Downing JR, Zwarthoff EC, Liu PP, Grosveld GC. MN1 overexpression is an important step in the development of inv(16) AML. Leukemia. 2007; 21:1679-1690.

17. Avvisati G, Lo-Coco F, Paoloni FP, Petti MC, Diverio D, Vignetti M, Latagliata R, Specchia G, Baccarani M, Di Bona E, Fioritoni G, Marmont F, Rambaldi A, et al. AIDA 0493 protocol for newly diagnosed acute promyelocytic leukemia: very long-term results and role of maintenance. Blood. 2011; 117:4716-4725. 